\title{
Changes in the Expression of Avian $\beta$-defensins (AvBDs) and Proinflammatory Cytokines and Localization of AvBD2 in the Intestine of Broiler Embryos and Chicks during Growth
}

\author{
Takumi Terada ${ }^{1}$, Takahiro Nii ${ }^{1,2}$, Naoki Isobe ${ }^{1,2}$ and Yukinori Yoshimura ${ }^{1,2}$ \\ ${ }^{1}$ Graduate School of Biosphere Science, Hiroshima University, Higashi-Hiroshima 739-8528, Japan \\ ${ }^{2}$ Research Center for Animal Science, Hiroshima University, Higashi-Hiroshima 739-8528, Japan
}

\begin{abstract}
The aim of this study was to determine the changes in the expression of avian $\beta$-defensins (AvBDs) and proinflammatory cytokines and localization of AvBD2 in the intestine of broiler embryos and chicks during growth. The ileum and cecum of embryonic day 19 (ED19) and of day-old (D0) and 7-day-old (D7) chicks were collected. Gene expression levels of 10 AvBDs $(A v B D 1-8,10$, and 12$)$ and proinflammatory cytokines $(I L-1 \beta,-6$, and -8$)$ were analyzed using real-time PCR, and the localization of AvBD2 was examined by immunohistochemistry. Gene expression levels of $A v B D 1,2,6$, and 7 in the ileum and of $A v B D 1$ and 4 in the cecum were higher on ED19 than on D7. The expression of $A v B D 10$ in the ileum was higher on D0 than on ED19, whereas the expression levels of $A v B D 8$ and 10 in the cecum were higher on D0 than on ED19, and that of $A v B D 10$ decreased on D7. The expression levels of $I L-1 \beta,-6$, and -8 in the ileum were higher on D7 than on ED19. The expression levels of $I L-1 \beta,-6$, and -8 in the cecum were higher on D0 than on ED19, and that of $I L-1 \beta$ and -6 declined on D7. AvBD2-positive cells were localized in the lamina propria beneath epithelial cells of villi and crypts. The number of positive cells in the cecum mucosa was greater on D0 than on ED19 and D7. In conclusion, we suggest that AvBDs are expressed in the ileum and cecum of embryos and chicks at high levels before or just after hatching and decrease by D7. The expression of proinflammatory cytokines in the ileum increases with growth until D7, but is the highest in the cecum around hatching. These AvBDs and proinflammatory cytokines may play roles in host defense in the intestinal mucosa of embryos and neonatal chicks.
\end{abstract}

Key words: avian $\beta$-defensin, broiler embryo and chick, innate immunity, intestine, proinflammatory cytokines

J. Poult. Sci., 55: 280-287, 2018

\section{Introduction}

The intestine is one of the organs that have a high risk of infection by pathogenic agents. Numerous microbes colonize the cecum soon after hatching (Crhanova et al., 2011). The mucosal innate immune system plays an essential role in defense against microbial infections (Smith and Beal, 2008). The gut-associated lymphoid tissues contain functionally immature $\mathrm{T}$ and $\mathrm{B}$ cells at the time of hatching, and their functionality is attained during the first 2 weeks post hatching (Bar-Shira et al., 2003). Thus, the adaptive immune system of the neonate is relatively immature, and maternal antibodies in the yolk transported to the chick are believed to play a role in protection against infection (Kovacs-Nolam

Received: February 12, 2018, Accepted: March 14, 2018

Released Online Advance Publication: April 25, 2018

Correspondence: Dr. Yukinori Yoshimura, Graduate School of Biosphere Science, Hiroshima University, Higashi-Hiroshima 739-8528, Japan.

(E-mail: yyosimu@hiroshima-u.ac.jp) and Mine, 2012). As it is generally accepted that the innate immune system develops at an early phase, it may play a primary role in the defense against infection in the embryo and chick.

Defensins are factors involved in innate immunity that has antimicrobial activity against a variety of microbes, including Gram-negative and -positive bacteria, enveloped viruses, and fungi (Ganz, 2003). In the chicken, fourteen avian $\beta$-defensins (AvBD1 to AvBD14) have been identified (Lynn et al., 2007). Lee et al. (2016) reported the antimicrobial activity of AvBD2-4, 6, 7, 11, and 13 against E. coli. Yacoub et al. (2015) reported that AvBD4 and 10 have antimicrobial activities against various bacteria and fungi. AvBD genes are expressed in the intestine, and are upregulated by Salmonella infection (Houng et al., 2012). Proinflammatory cytokines also play essential roles in immunomodulation and inflammation (Antonopoulos et al., 2017). Interleukin (IL)$1 \beta$ induces the expression of chemokines and nitric oxide, and the recruitment of immunocompetent cells in inflam- 
matory tissues (Dinarello, 2009). IL-6 is a multifunctional cytokine that regulates antibody synthesis (Chomarat et al., 2000). We have reported that IL- $1 \beta$ and IL-6 alsoupregulate the expression of AvBDs in the oviduct (Sonoda et al., 2013). IL-8 is the chemokine that recruits leukocytes (Rychlik et al., 2014).

As AvBDs are members of the innate immune system, and ILs are immunomodulators, they may both play pivotal roles in the intestinal defense system in young chicks. Crhanova et al. (2011) reported that the expression levels of $A v B D 1,2$, 4 , and 6 as well as of $I L-1 \beta$ and $I L-8$ in the cecum were higher during the first 3 days of life and decreased as of day 4. However, it remains unknown whether changes in AvBDs and cytokine expression occur in other intestinal segments. Cuperus et al. (2016) reported that AvBD9 is predominantly expressed in embryonic cells throughout the intestine. They also showed that an AvBD9 immuno-positive area was stable during development, except that the positive area in the jejunum was larger on day 1 post hatching than in embryos or 4-day-old chicks. However, the localization of other AvBDs, and their changes during chick growth, are unknown. The aim of this study was to determine the changes in the expression of $A v B D s$ and $I L-1 \beta,-6$, and -8 , and the localization of AvBD2 in the ileum and cecum in broiler embryos and chicks during their growth. We focused on the immunolocalization of AvBD2, because the antibody to AvBD2 was the best antibody that was raised in our laboratory to identify AvBDs in the intestine.

\section{Materials and Methods}

\section{Experimental Birds and Tissue Collection}

Fertilized eggs and male day-old chicks of broiler (Chunky) were obtained from a local hatchery (Fukuda Poultry, Okayama, Japan). The eggs were incubated in a moisturized incubator for 19 days. Chicks were maintained in a brooding room with a lighting schedule of $23 \mathrm{~h}$ light: $1 \mathrm{~h}$ dark for 7 days. The chicks were given a commercial starter diet (Nihon Nosan Kogyo Co. Ltd., Yokohama, Japan) and water ad libitum. Embryos at day 19 (ED19), day-old (before feeding; D0) and 7-day-old chicks (D7) were euthanized using carbon dioxide, and their ileums and cecums were collected ( $n=5$ each). This study was approved by the Hiroshima University Animal Research Committee (No. C1516).

RNA Isolation and Reverse Transcription PCR (RT-PCR)

RNA samples were extracted from the ileum and cecum using Sepazol RNA I Super (Nacalai Tesque Inc., Kyoto, Japan) according to the manufacturer's directions. RNA was dissolved in $10 \mathrm{mM}$ Tris with $1 \mathrm{mM}$ EDTA ( $\mathrm{pH} 8.0)$ and stored at $-80^{\circ} \mathrm{C}$ until use.

The RNA was treated with $1 \mathrm{U}$ RQ1 RNase-free DNase (Promega Co, Madison, WI, USA) in a $10-\mu l$ reaction mixture $(10 \mu \mathrm{g}$ total RNA, $1 \times$ DNase buffer and $1 \mathrm{U}$ DNase $)$ on a programmable thermal controller (PTC-100; MJ Research, Waltham, MA, USA) programmed at $37^{\circ} \mathrm{C}$ for $30 \mathrm{~min}$ and then at $65^{\circ} \mathrm{C}$ for $10 \mathrm{~min}$. The reaction was stopped with $1 \mathrm{U}$ RQ1 DNase Stop Solution (Promega Corporation, Madison,
USA). The concentration of RNA was measured using a NanoDrop Lite instrument (Thermo Fisher Scientific, Waltham, WV, USA). The RNA was then reverse-transcribed using ReverTra Ace (Toyobo Co. Ltd., Osaka, Japan) according to the manufacturer's instructions. The reaction mixture $(10 \mu l)$ consisted of $0.5 \mu \mathrm{g}$ total RNA, $1 \times$ reverse transcription buffer (Toyobo Co. Ltd.), $1 \mu \mathrm{M}$ deoxyribonucleotide triphosphate (dNTP) mixture (Toyobo Co. Ltd.), 5 U RNase inhibitor (Toyobo Co. Ltd.), $0.25 \mu \mathrm{g}$ of oligo(dT)20 (Toyobo Co. Ltd.), and $50 \mathrm{U}$ ReverTra Ace. Reverse transcription was carried out at $42^{\circ} \mathrm{C}$ for $30 \mathrm{~min}$, followed by heat inactivation at $99^{\circ} \mathrm{C}$ for $5 \mathrm{~min}$, in a programmable thermal controller (PTC-100; MJ Research). The cDNA samples were stored at $-20^{\circ} \mathrm{C}$ until use.

\section{Real-time PCR}

Real-time PCR was carried out using the AriaMix Realtime PCR system (Agilent Technologies Japan Ltd., Tokyo, Japan). The reaction mixture $(10 \mu l)$ consisted of $0.5 \mu l$ cDNA, $1 \times$ Brilliant III SYBR Green QPCR Mix (Agilent Technologies Japan Ltd.), $0.25 \mu \mathrm{M}$ of each primer, and water. The primers used for PCR are listed in Table 1. Two thermal protocols (two- or three-step) were used for PCR, depending on the target gene. For the two-step protocol, thermal cycles were as follows: 50 cycles of $95^{\circ} \mathrm{C}$ for $5 \mathrm{~s}$ and $62^{\circ} \mathrm{C}(A v B D 2$ to $4,6,12, I L-1 \beta,-8$, and $R P S 17)$ or $63^{\circ} \mathrm{C}$ $(A v B D 5,10$, and $I L-6)$ for $10 \mathrm{~s}$. For the three-step protocol, we used 50 cycles of $95^{\circ} \mathrm{C}$ for $5 \mathrm{~s}, 55^{\circ} \mathrm{C}(A v B D 1$ and 7$)$ or $58^{\circ} \mathrm{C}(A v B D 8)$, and $72^{\circ} \mathrm{C}$ for $10 \mathrm{~s}$. Because expression of $A v B D 9,11,13$, and 14 was not detected by real-time PCR in a preliminary analysis, these genes were not further examined (data not shown). Real-time PCR data were analyzed using the $2^{-\Delta \Delta \mathrm{CT}}$ method to calculate the relative levels of gene expression in each sample, and were expressed as ratios in relation to the housekeeping gene RPS17 (Livak and Schmittgen, 2001). An RNA sample of ED19 was used as a standard sample.

\section{Immunohistochemistry}

Anti-serum to AvBD2 was raised by immunization of rabbits with keyhole limpet hemocyanin-conjugated synthetic peptide (Medical \& Biological LAB, Nagoya, Japan). The sequence of the synthetic peptide was CPSHLIKVGS. The antibody was purified using HiTrap ${ }^{\mathrm{TM}}$ NHS-activated HP conjugated with synthetic peptide (GE Healthcare Japan, Tokyo, Japan).

The ileum and cecum were fixed in $10 \%(\mathrm{v} / \mathrm{v})$ formalin in phosphate-buffered saline (PBS), and processed for paraffin sectioning, at $4 \mu \mathrm{m}$ thickness. The sections were deparaffinized and then autoclaved at $110^{\circ} \mathrm{C}$ for $15 \mathrm{~min}$ in $10 \mathrm{mM}$ Tris with $1 \mathrm{mM}$ EDTA ( $\mathrm{pH} 10)$ for antigen retrieval. After cooling and washing with PBS (5 min, 3 times), endogenous peroxidases were quenched by incubation in $0.3 \%(\mathrm{v} / \mathrm{v}) \mathrm{H}_{2} \mathrm{O}_{2}$ in methanol for $10 \mathrm{~min}$. After washing with PBS (5 min, 3 times), the sections were incubated with $5 \%(\mathrm{w} / \mathrm{v})$ blocking regent (Roche Co., Basel, Switzerland) for $1 \mathrm{~h}$ to block nonspecific binding, followed by incubation with an antibody specific to AvBD2 diluted at $5 \mu \mathrm{g} / \mathrm{m} l$ in PBS for $16 \mathrm{~h}$. The sections were then washed in PBS (5 min, 3 times), and 
Table 1. PCR primers used for profiling of AvBDs and IL-1 $\beta,-6$ and $\mathbf{- 8}$

\begin{tabular}{|c|c|c|}
\hline Gene & sequences $\left(5^{\prime}-3^{\prime}\right)$ & Accession No. (Reference) \\
\hline \multirow[t]{2}{*}{$A v B D 1$} & F GATCCTCCCAGGCTCTAGGAAG & \multirow[t]{2}{*}{ NM_204993 (a) } \\
\hline & $\begin{array}{l}\text { R GCCCCATATTCTTTTGC } \\
\text { F GTTCTGTAAAGGAGGGTCCTGCCAC }\end{array}$ & \\
\hline$A v B D 2$ & R ACTCTACAACACAAAACATATTGC & NM_001201399 (b) \\
\hline \multirow{2}{*}{$A v B D 3$} & F CCACTCAGTGCAGAATAAGAG & \multirow{2}{*}{ NM_204650 (c) } \\
\hline & R AATTCAGGGCATCAACCTC & \\
\hline \multirow{2}{*}{$A v B D 4$} & F ATCGTGCTCCTCTTTGTGGCAGTTCA & \multirow{2}{*}{ NM_001001610 (b) } \\
\hline & R TACAACCATCTACAGCAAGAATACT & \\
\hline \multirow{2}{*}{$A v B D 5$} & F GCTGTCCCTTGCTCGAGGATT & \multirow{2}{*}{ NM_001001611 (b) } \\
\hline & R GGAATACCATCGGCTCCGGC & \\
\hline \multirow{2}{*}{$A v B D 6$} & F GATCCTTTACCTGCTGCTGTCT & \multirow{2}{*}{ NM_001001612 (b) } \\
\hline & R TCCTCACACAGCAAGATTTTAGTC & \\
\hline \multirow{2}{*}{$A v B D 7$} & F ACCTGCTGCTGTCTGTCCTC & \multirow{2}{*}{ NM_001001194 (a) } \\
\hline & R TGCACAGCAAGAGCCTATTC & \\
\hline \multirow{2}{*}{$A v B D 8$} & F TTCTCCTCACTGTGCTCCAA & \multirow{2}{*}{ NM_001001194 (a) } \\
\hline & R AAGGCTCTGGTATGGAGGTG & \\
\hline \multirow{2}{*}{$A v B D 9$} & F GCTTACAGCCAAGAAGACGCT & \multirow{2}{*}{ NM_001001194 (a) } \\
\hline & R GGAGCTAGGTGCCCATTTGCA & \\
\hline \multirow{2}{*}{$A v B D 10$} & F TGGGGCACGCAGTCCACAAC & \multirow{2}{*}{ NM_001001609 (c) } \\
\hline & R CATGCCCCAGCACGGCAGAA & \\
\hline \multirow{2}{*}{$A v B D 11$} & F ACTGCATCCGTTCCAAAGTCTG & \multirow{2}{*}{ NM_001001617 (b) } \\
\hline & R TCGGGCAGCTTCTCTACAAC & \\
\hline \multirow{2}{*}{$A v B D 12$} & F GGAACCTTTGTTTCGTGTTCA & \multirow{2}{*}{ NM_001001194 (a) } \\
\hline & R GAGAATGACGGGTTCAAAGC & \\
\hline \multirow{2}{*}{$A v B D 13$} & F GATCCTCCAGCTGCTCCTTG & \multirow{2}{*}{ NM_001001780 (a) } \\
\hline & R AGTGGCCATGGTTGTTCCT & \\
\hline \multirow{2}{*}{$A v B D 14$} & F CATATTCCTCCTGTTTCTTGTTCTC & \multirow{2}{*}{ NM_001001620 (b) } \\
\hline & R GCCAGTCCATTGTAGCAGGT & \\
\hline \multirow{2}{*}{$I L-1 \beta$} & F ACTGGGCATCAAGGGCTA & \multirow{2}{*}{ NM_204524 (d) } \\
\hline & R GGTAGAAGATGAAGCGGGTC & \\
\hline$J L-6$ & F AGAAATCCCTCCTCGCCAAT & NM 204628 (e) \\
\hline$I L-6$ & R AAATAGCGAACGGCCCTCA & NIM_204628 (e) \\
\hline LL-8 & F CTGTCCTGGCCCTCCTCCTGGTT & NM 001167719 (f) \\
\hline $1 L-\varnothing$ & R TGGCGTCAGCTTCACATCTTG & NM_00116//19 (1) \\
\hline$R P S 17$ & F AAGCTGCAGGAGGAGGAGAGG & NM 204217 (е) \\
\hline КГST & R GGTTGGACAGGCTGCCGAAGT & NIV1_20421/(C) \\
\hline
\end{tabular}

References for each primer are as follows; $\mathrm{a}=\mathrm{Xu}$ et al. (2015), $\mathrm{b}=$ Subedi et al. (2007), $\mathrm{c}=$ Ebers et al. (2009), $\mathrm{d}=$ Zang et al. (2012), $\mathrm{e}=$ Nii et al. (2011), $\mathrm{f}=$ Sundaresan et al. (2007).

incubated with biotin-conjugated anti-rabbit $\operatorname{IgG}(1: 200)$ and avidin-biotin-peroxidase complex (1:50) for $1 \mathrm{~h}$ each using a VECTASTAIN ABC kit (Vector Labs, Inc., Burlingame, CA, USA). The immunoreaction products were visualized using a reaction mixture of $0.02 \%(\mathrm{w} / \mathrm{v}) 3,3^{\prime}$-diaminobenzidine- $4 \mathrm{HCl}$ and $0.05 \%(\mathrm{v} / \mathrm{v}) \mathrm{H}_{2} \mathrm{O}_{2}$, and then, the sections were counterstained with hematoxylin.

Negative control staining by replacing the primary AvDB2 antibody with absorbed antibody was used to confirm antibody specificity. The absorbed antibody was prepared by incubating $5 \mu \mathrm{g}$ AvBD2 antibody and $25 \mu \mathrm{g}$ synthetic AvBD2 peptide in $1 \mathrm{ml}$ of PBS at $4^{\circ} \mathrm{C}$ for $16 \mathrm{~h}$.

\section{Image Analysis}

Sections were examined under a light microscope connected to an image analysis software (NIS-Elements, Nikon, Tokyo, Japan). AvBD2-positive cells in the whole area of mucosal tissue (mucosal epithelium, crypts, and lamia propria) on each section of the ileum and cecum were counted, followed by calculation of positive cell numbers in an area of $1 \times 10^{5} \mu \mathrm{m}^{2}$.

\section{Statistical Analysis}

All data are expressed as the mean \pm SEM. The significance of differences in gene expression levels and the AvBD2-positive cell counts among different growth stages were obtained using one-way ANOVA followed by Tukey's test, or the Kruskal-Wallis test followed by the Steel-Dwass test. Differences were considered significant when the $P$ value was $<0.05$.

\section{Results}

Figure 1 shows the changes in the expression of 10 AvBDs ( $A v B D 1-8,10$, and 12) in the ileum and cecum during em- 
(A) $A v B D 1$

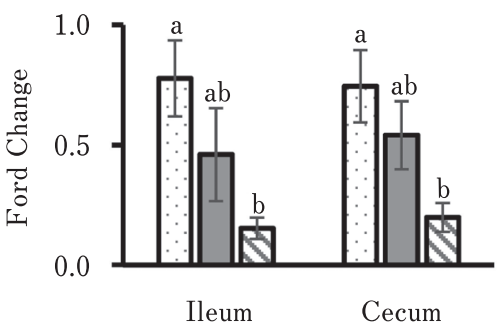

(C) $A v B D 3$

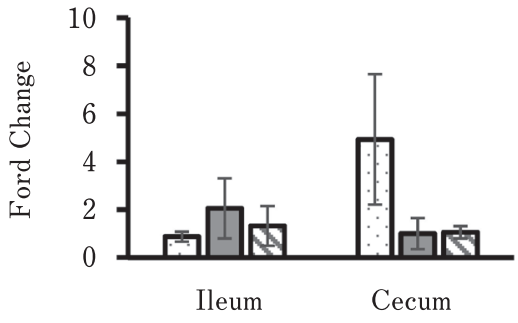

(E) $A v B D 5$

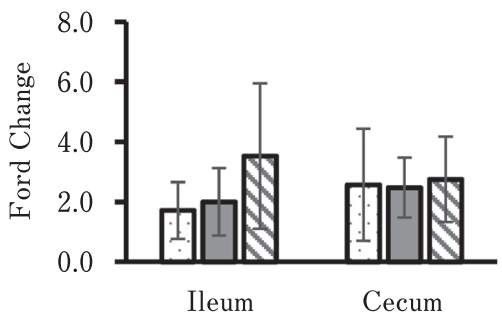

(G) $A v B D 7$

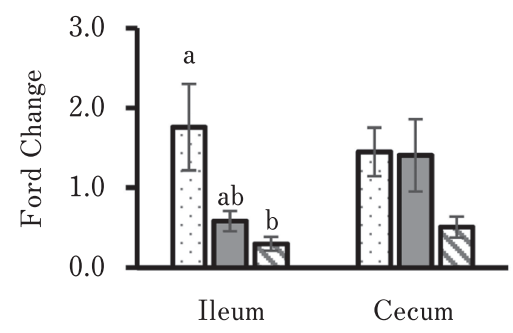

(I) AvBD10

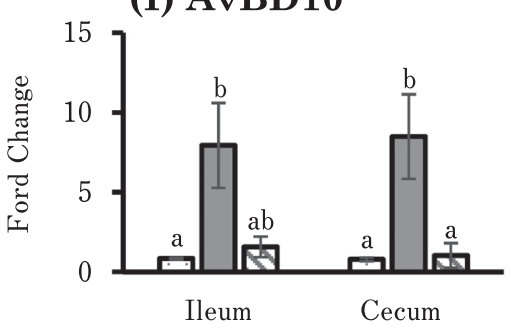

(B) $A v B D 2$

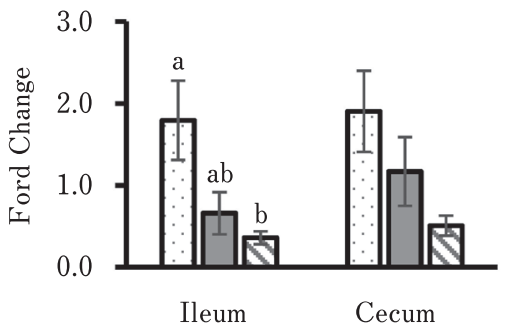

(D) $A v B D 4$

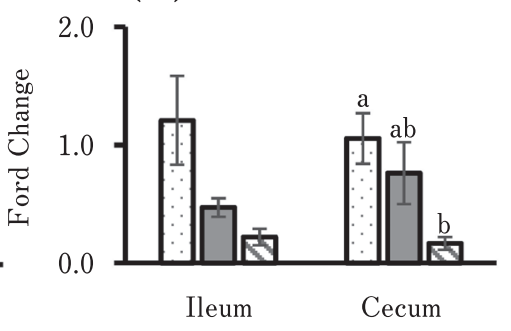

(F) $A v B D 6$

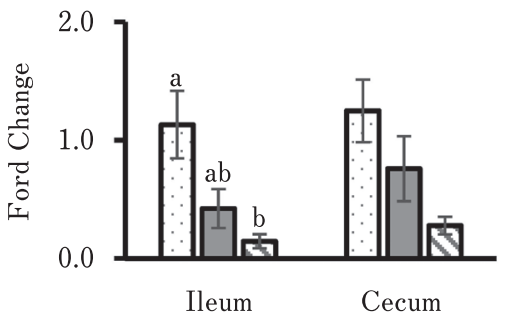

(H) $A v B D 8$
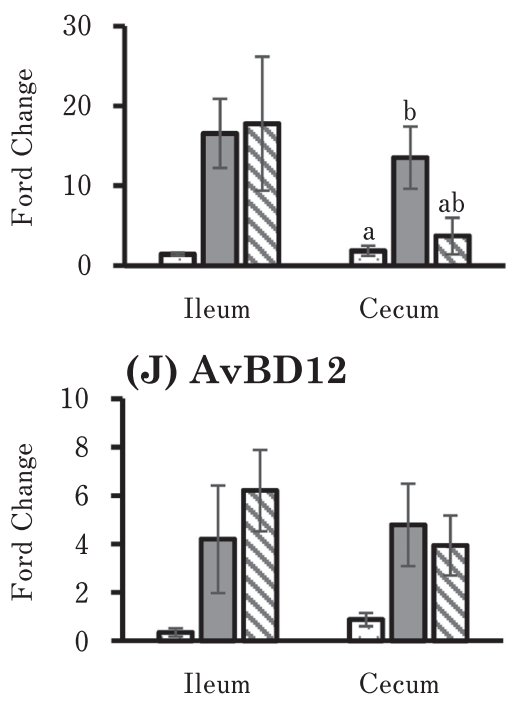

Fig. 1. Changes in the gene expression levels of $A v B D 1-8,10$, and 12 in the ileum and cecum during the growth of embryos and chicks. Values are the mean \pm SEM $(n=5)$ of fold changes in expression.

${ }^{\mathrm{a}, \mathrm{b}}$ Values with different letters are significantly different among ED19, D0 and D7 within the ileum or cecum $(P<0.05)$. 


\section{$\square \mathrm{ED} 19 \square \mathrm{D} 0 \quad \boldsymbol{\nabla} \mathrm{D} 7$}

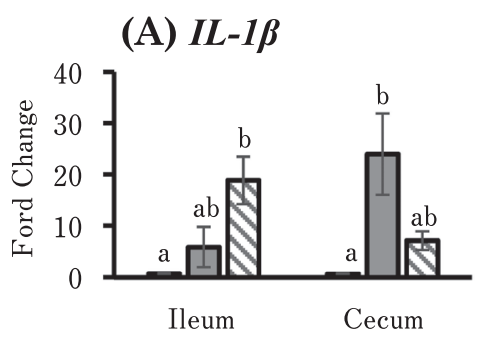

(B) $I L-6$
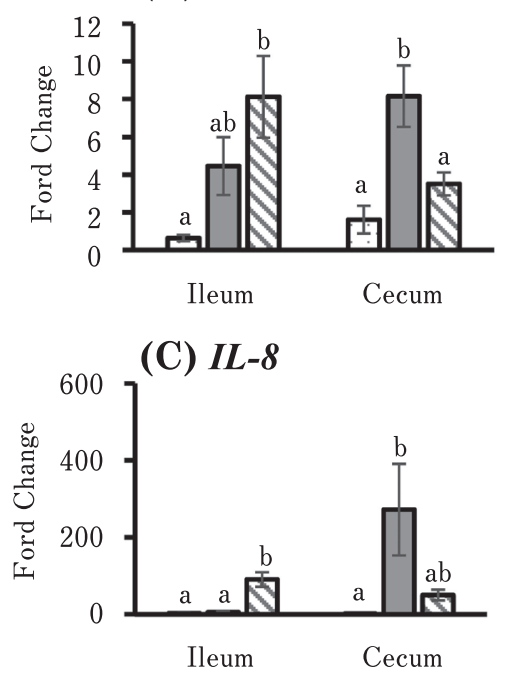

Fig. 2. Changes in the gene expression levels of $I L-1 \beta$, -6 , and -8 in the ileum and cecum during the growth of embryos and chicks. The values are the mean $\pm \operatorname{SEM}(n=$ 5) of fold changes in expression. ${ }^{\mathrm{a}, \mathrm{b}}$ Values with different letters are significantly different among ED19, D0 and D7 within the ileum or cecum $(P<0.05)$.

bryo and chick growth. In the ileum, the expression levels of $A v B D 1,2,6$, and 7 were significantly lower on $\mathrm{D} 7$ than on ED19 (Fig. 1A, B, F, and G). In contrast, the expression level of $A v B D 10$ on D0 was significantly ( $\sim 7$-fold) higher than that on ED19 (Fig. 1I). However, the expression levels of $A v B D 3,4,5,8$, and 12 were not significantly different among ED19, D0, and D7 (Fig. $1 \mathrm{C}-\mathrm{E}, \mathrm{H}$, and J). In the cecum, the expression levels of $A v B D 1$ and 4 on $\mathrm{D} 7$ were significantly lower than those at ED19 (Fig. 1A and D). The expression levels of $A v B D 8$ and 10 in the cecum were significantly higher on D0 than on ED19, and that of $A v B D 10$ was decreased on D7 (Fig. 1H and I). However, the expression levels of $A v B D 2,3,5,6,7$, and 12 did not significantly change during growth from ED19 to D7 (Fig. 1B, C, E, F, and $\mathrm{G})$.

Figure 2 shows the changes in the expression of $I L-1 \beta,-6$, and -8 in the ileum and cecum during growth. The expression levels of $I L-1 \beta,-6$, and -8 in the ileum on D7 were approximately 16-, 7-, and 90-fold higher, respectively, than those on ED19 (Fig. 2A to C). The expression levels of $I L-$
$1 \beta,-6$, and -8 in the cecum were approximately $23-, 5-$, and 270- fold higher on D0 than on ED19, and expression of $I L$ $1 \beta$ and -6 declined on D7 (Fig. 2A to C).

Figure 3 shows the localization of AvBD2-positive cells in the ileum and cecum on ED19, D0, and D7. The AvBD2positive cells were localized in the lamina propria, beneath the epithelium of the villi and crypts in both the ileum (Fig. 3A, C, E) and the cecum (Fig. 3B, D, F) at all stages. A few positive cells were localized in the epithelium of villi and crypts (Fig. 3D). The localization profiles of AvBD2 in the ileum and cecum were not different among the growth stages evaluated. Figure 4 shows the frequencies of AvBD2-positive cells (cell number in an area of $1 \times 10^{5} \mu \mathrm{m}^{2}$ ) in mucosal tissues. In the cecum, the AvBD2-positive cell frequency was significantly higher on D0 than on ED19 or D7. In contract, their frequency in the ileum was not significantly different among the growth stages. Negative control staining using absorbed antibody did not reveal any reaction products in either the ileum or the cecum (Fig. 3G, H).

\section{Discussion}

We report the changes in the expression levels of AvBD genes and $I L-1 \beta,-6$, and -8 and the localization of immunoreactive AvBD2 in the ileum and cecum of chicken embryos and chicks during their early phase of growth, i.e., ED19, D0, and D7. Major findings were that (1) AvBD expression levels showed 3 distinct profiles: highest on D0 ( $A v B D 8$ and 10), decreased after hatching ( $A v B D 1,2,4,6$, and 7$)$, and no changes $(A v B D 3,5$, and 12) in the ileum or cecum; (2) the $I L-1 \beta$, -6 and -8 expression levels were increased on D7 in the ileum and on D0 in the cecum; and (3) the AvBD2-positive cells were localized in the lamina propria of the ileum and cecum, and their frequency in the cecum was higher on D0 than on ED19 or on D7.

The results of the current study showed that expression levels of $A v B D 1,2,6$, and 7 in the ileum, and of $A v B D 1$ and 4 in the cecum on D7 were decreased as compared to the levels on ED19. The highest expression levels of $A v B D 8$ and 10 were also found in the cecum on D0, and that of $A v B D 10$ declined by D7. These results, showing decreased AvBD expression with post-hatch growth, support the findings reported by Crhanova et al. (2011) that in the first three days of life, the chicken cecum was protected by increased expression of $A v B D 1,2,4$, and 6, the expression of which dropped as of day 4 of life. The current study showed that the decline in AvBD expression in the post-hatch period occurs not only in the cecum, but also in the ileum. Su et al. (2017) reported that Eimeria acervulina challenge resulted in the downregulation of $A v B D 1,6,10,11,12$, and 13 in the duodenum, and that Eimeria maxima challenge downregulated $A v B D 6,10$, and 11 in the duodenum and $A v B D 10$ in the jejunum. The complexity of microbiota gradually increases in the chick intestine, with the most dramatic development occurring during the first week of life (Crchanova et al., 2011). Accordingly, as observed in the current study, the expression of multiple AvBDs changed during the first week of life, and there seems to be an association between the 


\section{Ileum}
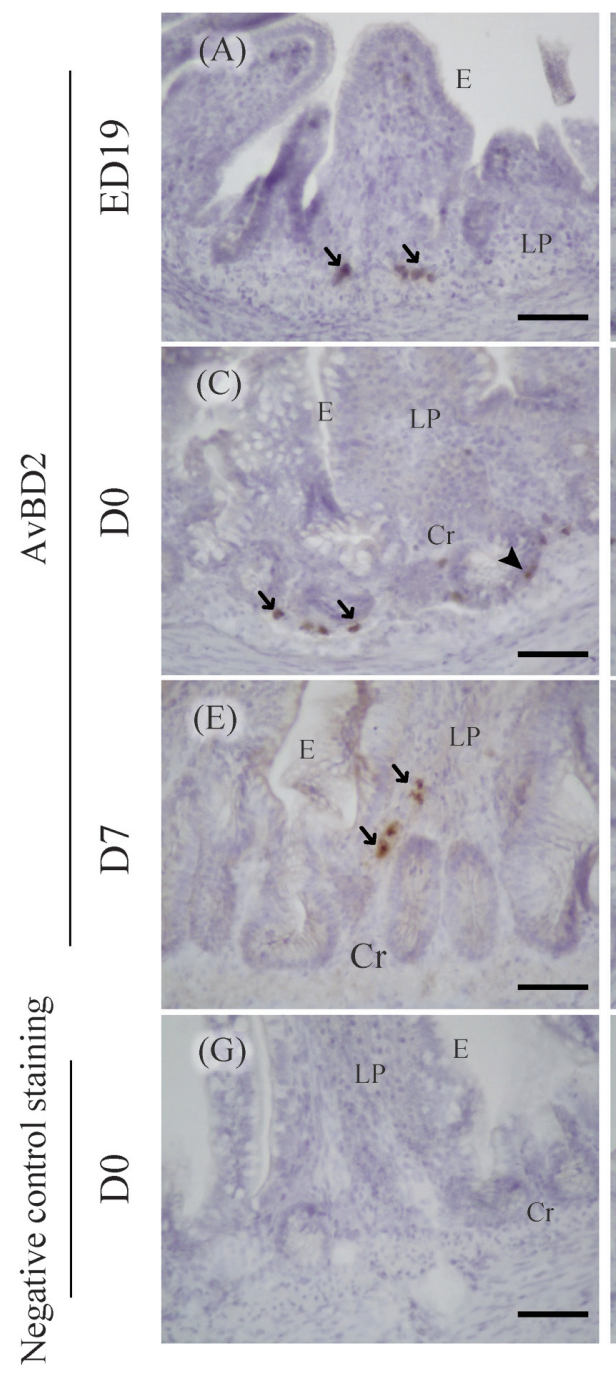
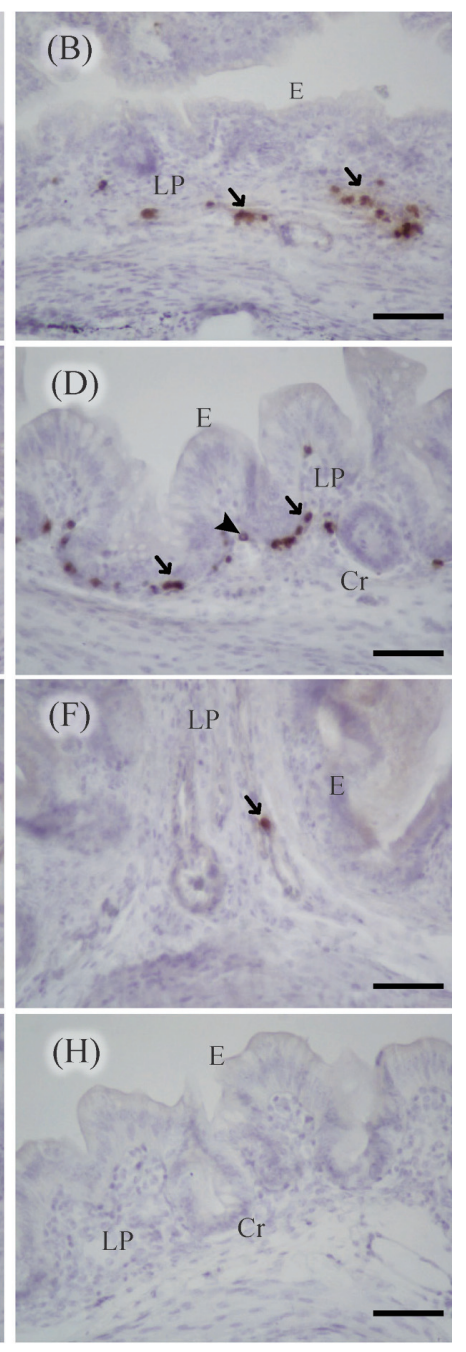

Fig. 3. Micrographs showing immunoreactive AvBD2 in the ileum and cecum on embryonic day 19 (ED19), and days 0 (D0) and 7 (D7) of the post-hatching period. AvBD2-immunoreactive cells are localized in the lamina propria beneath the epithelium in each tissue at all developmental stages (arrows). Some of the positive cells are localized in the epithelium of the villi and crypts (arrowheads). G and $\mathrm{H}$ show negative control staining using absorbed antibody. $\mathrm{E}=$ mucosal epithelium, $\mathrm{LP}=$ lamina propria, $\mathrm{Cr}=$ crypt. Scale bar $=50 \mu \mathrm{m}$.

colonization of natural or specific microbiota complexes and changes in AvBDs expressed during the early post-hatch period. However, challenge with specific microorganisms may cause an upregulation of AvBDs, as expression of $A v B D 8$ and 13 in the cecum of 21-day-old broiler chickens was upregulated by Eimeria maxima (Su et al., 2017), and expression of $A v B D 1,2,4$, and 6 was increased in the cecal tonsils of broiler chicks 3-5 days post infection by Salmonella Typhimurium, starting from 1 day of age (Akbari et al., 2008). The intestinal microbiota composition plays an im- portant role in the maintenance of gut health (Clavijo and Flórez, 2018). It is reported that AvBD1 is cytotoxic to $E$. coli and E. faecalis, and AvBD4 and 10 are cytotoxic to $S$. Typhimurium and E. coli (Yacoub et al., 2015). Lee et al. (2016) suggested that $E$. coli is killed by AvBD1-4, 6, 7, 9, 11,13 , and 14. Thus, AvBDs synthesized in the ileum and cecum may play roles in the regulation of the microbiota population.

Our results showed that the expression of $I L-1 \beta,-6$, and -8 was higher on D0 and D7 than on ED19 in the ileum and 


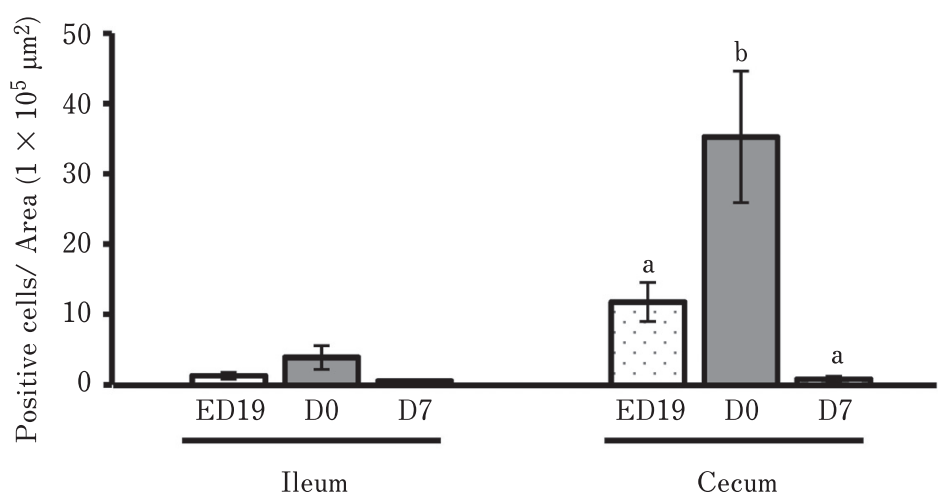

Fig. 4. Changes in the frequencies of AvBD2-positive cells in the mucosa of the ileum and cecum on embryonic day 19 (ED19), and days 0 (D0) and 7 (D7) of the post-hatching period. Values are the mean $\pm \operatorname{SEM}(n=5)$ of the number of positive cells in an area of $1 \times 10^{5}$ $\mu \mathrm{m}^{2} .{ }^{\mathrm{a}, \mathrm{b}}$ Values with different letters indicate significantly different within the cecum $(P<0.05)$.

cecum, respectively. Thus, the expression of these cytokines may be upregulated in association with the development of intestinal microflora. Crhanova et al. (2011) demonstrated that, for the first three days of life, the chicken cecum is protected by AvBDs, and this type of cecum protection was replaced from day 4 of life by normalization of the gut immune system developed by proinflammatory cytokines IL8 and IL-17. Although we have not examined the expression of $I L-17$, the higher levels of $I L-1 \beta, I L-6$, and $I L-8$ may suggest that the development of the mucosal immune system may be induced by these cytokines during the first week of life.

Our results showed that AvBD2-positive cells were localized in the lamina propria beneath the epithelium of villi and crypts. Cuperus et al. (2016) reported that AvBD9 was predominantly found in the enteroendocrine cells of the intestines. However, expression of AvBD1, 2, and 7 has been identified in the bone marrow tissue in chickens (Derache et al., 2009), suggesting that bone marrow-derived leukocytes are involved in synthesis of these AvBDs. Thus, the AvBD2-positive cells identified in this study may be the member of leucocytes derived from bone-marrow. The frequency of AvBD2-positive cells was increased on D0 compared to ED19 and was decreased on D7 in the cecum. These cells may play an important role in host defense at an early phase of the post-hatch period. Although $A v B D 2$ gene expression was higher on ED19, AvBD protein synthesis might be immature before hatching. The expression of the $I L-8$ chemokine was also higher on D0. We assume that IL8 may be one of the factors involved in the recruitment of AvBD2-positive cells in the cecum. Such cells may decrease in association with the development of the gut-acquired immune system. The frequency of AvBD2-positive cells in the ileum was relatively low and remained unchanged during the embryonic and post-hatch phases. The recruitment level of those cells may be unchallenged under healthy conditions in this particular tissue. The cecum retains intestinal internal contents for the longest period within the intestine, and microbiota of the cecum play roles in the digestion of substances rich in cellulose and others (Clavijo and Flórez, 2018). These differential characteristics in terms of microbiota and intestinal internal contents in the cecum may be considered when examining why changes in the AvBD2 cell population are different between the ileum and cecum.

In conclusion, we suggest that AvBDs are expressed in the ileum and cecum of the embryo and young chick, with levels being high before or right after hatching, and decreasing by D7. AvBD2-positive cells are likely recruited in the cecum around the hatching phase. The expression of proinflammatory cytokines increases with the growth of chicks till D7 in the ileum, but showed the highest levels around hatching in the cecum. These AvBDs and proinflammatory cytokines may play roles in the host defense in the intestinal mucosa of chick embryos and neonates.

\section{Acknowledgments}

This study was supported by a Grant-in-Aid for Scientific Research (B) from the Japan Society for the Promotion of Science (No. 17H03904) and by a grant from the Mishima Kaiun Memorial Foundation (Tokyo, Japan) awarded to YY.

\section{References}

Akbari MR, Haghighi HR, Chambers JR, Brisbin J, Read LR and Sharif S. Expression of antimicrobial peptides in cecal tonsils of chickens treated with probiotics and infected with Salmonella enterica Serovar Typhimurium. Clinical and Vaccine Immunology, 15: 1689-1693. 2008.

Antonopoulos AS, Papanikolaou E, Vogiatzi G, Oikonomou E and Tousoulis D. Anti-inflammatory agents in peripheral arterial disease. Current Opinion Pharmacology, 39: 1-8. 2017.

Bar-Shira E, Sklan D and Friedman A. Establishment of immune competence in the avian GALT during the immediate post- 
hatch period. Development \& Comparative Immunology, 27: 147-157. 2003.

Chomarat P, Banchereau J, Davoust J and Palucka AK. IL-6 switches the differentiation of monocytes from dendritic cells to macrophages. Nature Immunology, 1: 510-514. 2000.

Clavijo V and Flórez MJV. The gastrointestinal microbiome and its association with the control of pathogens in broiler chicken production: A review. Poultry Science, 97: 1006-1021. 2018.

Crhanova M, Hradecka H, Faldynova M, Matulova M, Havlickova $\mathrm{H}$, Sisak F and Rychlik I. Immune response of chicken gut to natural colonization by gut microflora and to Salmonella enterica serovar enteritidis infection. Infection and Immunity, 79: 2755-2763. 2011.

Cuperus T, van Dijk A, Dwars RM and Haagsman HP. Localization and developmental expression of two chicken host defense peptides: cathelicidin- 2 and avian $\beta$-defensin 9. Development and Comparative Immunology, 61: 48-59. 2016.

Derache C, Labas V, Aucagne V, Meudal H, Landon C, Delmas AF, Magallon T and Lalmanach AC. Primary structure and antibacterial activity of chicken bone marrow-derived betadefensins. Antimicrobial Agents and Chemotherapy, 53: 46474655. 2009.

Dinarello CA. Immunological and inflammatory functions of the interleukin-1 family. Annual Review of Immunology, 27: 519550. 2009.

Ebers KL, Zhang CY, Zhang MZ, Bailey RH and Zhang S. Transcriptional profiling avian beta-defensins in chicken oviduct epithelial cells before and after infection with Salmonella enterica serovar Enteritidis. BMC Microbiology, 9: 153. 2009.

Ganz T. Defensins: antimicrobial peptides of innate immunity. Nature Reviews Immunology, 3: 710-720. 2003.

Hong YH, Song W, Lee SH and Lillehoj HS. Differential gene expression profiles of $\beta$-defensins in the crop, intestine, and spleen using a necrotic enteritis model in 2 commercial broiler chicken lines. Poultry Science, 91: 1081-1088. 2012.

Kovacs-Nolan J and Mine Y. Egg yolk antibodies for passive immunity. Annual Review of Food Science and Technology, 3: 163-182. 2012.

Lee MO, Jang HJ, Rengaraj D, Yang SY, Han JY, Lamont SJ and Womack JE. Tissue expression and antibacterial activity of host defense peptides in chicken. BMC Veterinary Research, 12: 231. 2016.

Livak KJ and Schmittgen TD. Analysis of relative gene expression data using real-time quantitative PCR and the $2^{-\triangle \Delta C T}$ method. Methods, 25: 402-408. 2001.

Lynn DJ, Higgs R, Lloyd AT, O'Farrelly C, Hervé-Grépinet V, Nys
Y, Brinkman FS, Yu PL, Soulier A, Kaiser P, Zhang G and Lehrer RI. Avian beta-defensin nomenclature: a community proposed update. Immunology Letters, 15: 86-89. 2007.

Nii T, Sonoda Y, Isobe N and Yoshimura Y. Effects of lipopolysaccharide on the expression of proinflammatory cytokines and chemokines and the subsequent recruitment of immunocompetent cells in the oviduct of laying and molting hens. Poultry Science, 90: 2332-2341. 2011.

Rychlik I, Elsheimer-Matulova M and Kyrova K. Gene expression in the chicken caecum in response to infections with nontyphoid Salmonella. Veterinary Research, 45: 119. 2014.

Smith AL and Beal R. The avian enteric immune system in health and disease. In: Avian Immunology. Vol. 1. pp. 243-271. Elsevier. London. 2008.

Sonoda Y, Abdel Mageed AM, Isobe N and Yoshimura Y. Induction of avian $\beta$-defensins by $\mathrm{CpG}$ oligodeoxynucleotides and proinflammatory cytokines in hen vaginal cells in vitro. Reproduction, 145: 621-631. 2013.

Su S, Dwyer DM, Miska KB, Fetterer RH, Jenkins MC and Wong EA. Expression of host defense peptides in the intestine of Eimeria-challenged chickens. Poultry Science, 96: 2421-2427. 2017.

Subedi K, Isobe N, Nishibori M and Yoshimura Y. Changes in the expression of gallinacins, antimicrobial peptides, in ovarian follicles during follicular growth and in response to lipopolysaccharide in laying hens (Gallus domesticus). Reproduction, 133: 127-133. 2007.

Sundaresan NR, Anish D, Sastry KV, Saxena VK, Mohan J and Ahmed KA. Cytokines in reproductive remodeling of molting white leghorn hens. Journal of Reproductive Immunology, 73: 39-50. 2007.

Xu Y, Zhang T, Xu Q, Han Z, Liang S, Shao Y, Ma D and Liu S. Differential modulation of avian $\beta$-defensin and toll-like receptor expression in chickens infected with infectious bronchitis virus. Applied Microbiology Biotechnology, 99: 90119024. 2015.

Yacoub HA, Elazzazy AM, Abuzinadah OA, Al-Hejin AM, Mahmoud MM and Harakeh SM. Antimicrobial activities of chicken $\beta$ defensin (4 and 10) peptides against pathogenic bacteria and fungi. Frontiers in Cellular and Infection Microbiology, 5: 36. 2015.

Zang M, Nii T, Isobe N and Yoshimura Y. Expression of toll-like receptors and effects of lipopolysaccharide on the expression of proinflammatory cytokines and chemokine in the testis and epididymis of rooters. Poultry Science, 91: 1997-2003. 2012. 